\title{
The pulsation modes and masses of carbon-rich long period variables $\star, \star \star$
}

\author{
J. Bergeat, A. Knapik, and B. Rutily \\ Centre de Recherche Astronomique de Lyon (UMR 5574 du CNRS), Observatoire de Lyon, \\ 9 avenue Charles André, 69561 St-Genis-Laval cedex, France \\ Received 12 March 2002 / Accepted 15 March 2002
}

\begin{abstract}
Following our study of the carbon-rich giants in the HR diagram and of their luminosity function (Paper III), we investigate the pulsation data of the long period variables (LPVs) included in our sample. Pulsation modes (fundamental, overtone(s)) for carbon LPVs are identified in the period-radius diagram, making use of observed bi-periodicity in a small subsample of those stars, and of comparison to models. Mean pulsation masses are then deduced from theoretical PMR-relations, with due attention paid to a possible bias while averaging.

Mean (present) pulsation masses $\left(0.6-4.0 M_{\odot}\right)$ are found to increase along the group sequence HC5 to CV6, with still larger masses possibly associated with cool extreme CV7-objects with strong mass loss and thick circumstellar shells. This is consistent with the $0.8-4 M_{\odot}$ range of initial masses found in Paper III for the majority of carbon-rich giants affected by mass loss during their evolution. The pulsation masses found for a few HC-stars $\left(M \leq 0.8 M_{\odot}\right)$ are consistent with their low initial masses $\left(M_{\mathrm{i}} \lessgtr 1.1 M_{\odot}\right)$, as inferred from their thick disk membership (age $\simeq 11 \mathrm{Gyr}$ ?) and locus in the HR diagram. A mean pulsation mass of $\simeq 0.6 M_{\odot}$ is found for the three population II Cepheids in the sample. A mass-luminosity diagram is proposed for the Galactic carbon giants. The data from observations is found consistent with theoretical predictions from AGB modeling, specially the third dredge-up (TDU) through thermal pulses (TP) with a carbon star formation line (CSFL) for TP-AGB stars. It appears that the CV-giants are close to the tip and end of their evolutionary tracks in the TP-AGB of the HR diagram. It is confirmed that this end shifts toward lower effective temperatures and higher luminosities, with increasing masses.

It is shown that the $\mathrm{C} / \mathrm{O}$ abundance ratios do correlate with effective temperatures, according to three distinct distributions (halo $\mathrm{CH}$ stars, thick disk HC-stars, and thin disk CV-stars). The mean stellar density decreases along the HC5-CV7 sequence, while the surface gravity remains nearly constant at about 0.5 CGS unit $\left(\log g \simeq-0.3 ; 5 \times 10^{-3} \mathrm{SI}\right)$.

The nature of (thin disk) CV-stars as TP-AGB objects being confirmed, the discussion is focused on (thick disk) HC-stars since the origin of these old low-mass giants remains unclear. Unpredicted extra mixing on RGB and/or E-AGB is favored. Evolution from (old, low $\mathrm{O} / \mathrm{H}$ ) dwarf carbon stars is also considered since observations of metal-poor stars and recent calculations point to large supersolar $[\mathrm{C} / \mathrm{Fe}]$ ratios in Population III objects and contamination through rapid cycling in the interstellar medium.
\end{abstract}

Key words. stars: AGB and post-AGB - stars: carbon - stars: late-type - stars: fundamental parameters stars: Hertzsprung-Russell (HR) and C-M diagrams - stars: luminosity function, mass function

\section{Introduction}

In a companion paper (Bergeat et al. 2002b, hereafter Paper III) a general introduction was provided to the subject of fundamental quantities (mass, luminosity, radius, effective temperature etc.) for carbon-rich giants, and diagrams in which their evolution can be traced. Following the HIPPARCOS mission (ESA 1997), associating astrometric data and radial velocities, spectral energy distributions (SEDs) and photometric classification, and angular diameters from lunar occultations and

Send offprint requests to: J. Bergeat, e-mail: bergeat@obs.univ-lyon1.fr

* This research has made use of the Simbad database operated at CDS, Strasbourg, France.

$\star \star$ Partially based on data from the ESA HIPPARCOS astrometry satellite. interferometry, our knowledge of those complex phases of stellar evolution considerably improved (Bergeat et al. 2001 hereafter Paper I; Bergeat et al. 2002a hereafter Paper II, and references therein). In Paper III, it was shown that the luminosity function (LF) of the Galactic carbon-rich giants in the Sun vicinity exhibit two maximas corresponding respectively to (faint) HC-objects of the thick disk contaminated by halo $\mathrm{CH}$ stars, and (bright) CV-members of the thin disk. The LF of the former giants appears similar to that of the Galactic bulge reinforcing the idea of a dynamical connection between Bulge and thick disk, while the LF of the latter seems similar to that of the Large Magellanic Cloud (LMC). The HR diagram of nearly 370 Galactic carbon and BaII giants, and related objects was constructed and discussed. The ${ }^{99} \mathrm{Tc}$-rich stars were found to be (bright) CV-giants as expected, which is consistent with the third dredge-up (TDU) scenario. A similar result was 
already obtained for bright (intrinsic) S stars (Van Eck et al. 1998). The ${ }^{13} \mathrm{C}$-rich objects (J-stars) are on the contrary nearly uniformly distributed within $\mathrm{HC}$ and $\mathrm{CV}$-giants in the HR diagram. From comparison to predicted tracks for stellar evolution with various $(Z)$ abundances in heavy elements, a range of initial masses of 0.8 up to $4 M_{\odot}$ was established for the majority of carbon-rich giants.

From periods of pulsation of long period variables (LPVs), the present masses can be estimated ("pulsation masses"). This requires identification in the observations of the various pulsation modes, and comparison of data to theoretical periodmass-radius (PMR) relations. Such a study has already been attempted for oxygen-rich LPVs or on samples including only a few carbon variables, making use of linear models (e.g. Barthès 1998 and references therein). Groenewegen \& de Jong (1994) studied the pulsation modes of the LPVs in the LMC, including carbon-rich objects. They concluded that most of them should pulsate in the fundamental mode. The derived masses fall in the ranges of the low mass stars (LMS) and intermediate mass stars (IMS).

The pulsation mode of carbon LPVs is analyzed in the period-radius (PR) diagram (Sect. 2), and the multiperiodicity of part of the stars in our sample, is exploited to confirm the splitting of the PR-diagram into two portions for fundamental and (presumably first) overtone modes respectively. The (present) "pulsation" masses are estimated (Sect. 3), applying the theoretical PMR-relations to the observed data. The results are displayed in a mass-luminosity diagram, where they are confronted to the prediction of stellar evolution calculations (Sect. 4). The carbon to oxygen abundance ratio $(\mathrm{C} / \mathrm{O})$ does exhibit distinct behaviors vs. effective temperature (Sect. 5), for three populations found in the Sun vicinity. The main results are finally summarized and discussed (Sect. 6), with special emphasis on luminosities, initial and present masses, evolutionary status, mean densities and surface gravities.

\section{The pulsation modes of carbon LPVs}

\subsection{Introduction}

The long period variables (LPVs) are cool giants whose pulsation properties are similar to those of their oxygen-rich analogues. A few Population II Cepheids (three stars with two confirmed carbon-rich Cepheids, are included in our sample: see Sect. 7.6 of Paper III). We have shown in Sect. 5 of Paper III that mean luminosities and photospheric radii are, on average, increasingly larger along a sequence of increasing variability. No discontinuity in the obtained data could be observed. Here, we concentrate on variables of the classes SRb and SRa (semi-regulars) and M (Miras) with carbon-rich atmospheres and known periods. Many SRb-variables or stars classified SR or SR:, are not included in our study in the absence of reliable periods. Conversely, new recent data of higher accuracy, mainly from HIPPARCOS, showed that a few stars previously classified as Lb (irregulars), actually display light curves of SRb (semi-regulars) variables.

As it is the case for classical Cepheids, period-luminosity relations, then refined as period-luminosity-radius relations
(PLR), have been established. A period-luminosity relation was discovered for the Miras (essentially oxygen-rich) of the LMC (Glass \& Lloyd Evans 1981). It was subsequently improved by Feast (1984) and Feast et al. (1989), who considered the relation as a relatively tight one, the dispersion being of only \pm 0.15 mag. The relation was slightly revised by Groenewegen \& Whitelock (1996) for carbon Miras, with a zero-point based on the Galactic star UV Aur, a member of a double system (see also van Leeuwen et al. 1997).

A crucial question is that of the mode of pulsation. Concerning oxygen-rich LPVs, arguments in favor of the first overtone were given by Haniff et al. (1995), although the fundamental mode may often be seriously considered in some cases. Barthès (1998) modeled a sample of 22 Miras and found that a significant proportion of them pulsate in the fundamental mode. Bergeat et al. (1998) studied the period-luminosity relation of Galactic carbon variables from HIPPARCOS data, making use of $M_{\mathrm{K}}$, the absolute magnitude in the near infrared, and compared it with its analogue in the LMC. The periodluminosity relation they found for Galactic carbon variables ("Sample 1") is very close to the relation of Reid et al. (1995) for their LMC analogues. In both systems however, brighter carbon variables are observed at short periods ("Sample 2") and underluminous stars ("Sample 3") are found at long periods. Following Wood \& Sebo (1996), Bergeat et al. (1998) suggested that stars in Sample 2 are overtone pulsators while those in Sample 1 might be pulsating in the fundamental mode. The status of Sample 3 underluminous variables remained unclear, but they should be pulsating in the same (fundamental) mode as Sample 1 stars. Mode identification was provided in a period-radius diagram, for some oxygen-rich variables, by van Leeuwen et al. (1997). A similar approach is applied to the carbon-rich variables in Sect. 2.2.

Theoretical calculations of PLR relations were published for oxygen-rich LPVs (Fox \& Wood 1982; Wood et al. 1983; Wood 1990, and references therein). Unfortunately, no calculation is available for carbon-rich opacities. Luminosities and photospheric radii predicted for overtone models are larger than those for fundamental models, for a given period of pulsation. The calculations of pulsations in stellar envelopes are usually linear ones, with or without the assumption of adiabaticity. In many cases, when the fundamental mode predominates, the model diverges. This mode was interpreted as a source of violent processes of strong mass loss (Wood 1974; Tuchman et al. 1979), and considered as not reproducing the actual observations which imply more steady mass loss. The modeled period of the first overtone and the velocity amplitude in the atmosphere often fall short of observed values. Ya'ari \& Tuchman $(1996,1999)$ operated non-linear dynamical simulations which follow up on the development of oscillations due to initial perturbations chosen in various ways. The calculations which spread over 600 years, show that the models end up pulsating in a fundamental mode whose period is shorter than the period of the initial static model, after having pulsated e.g. for more than 150 years in the first overtone. This is the consequence of a thermal adjustment in the envelope (Ya' ari \& Tuchman 1996). Models also exist which remain in the first overtone (Ya'ari 1999). Barthès (1998) however argued that dynamical models 


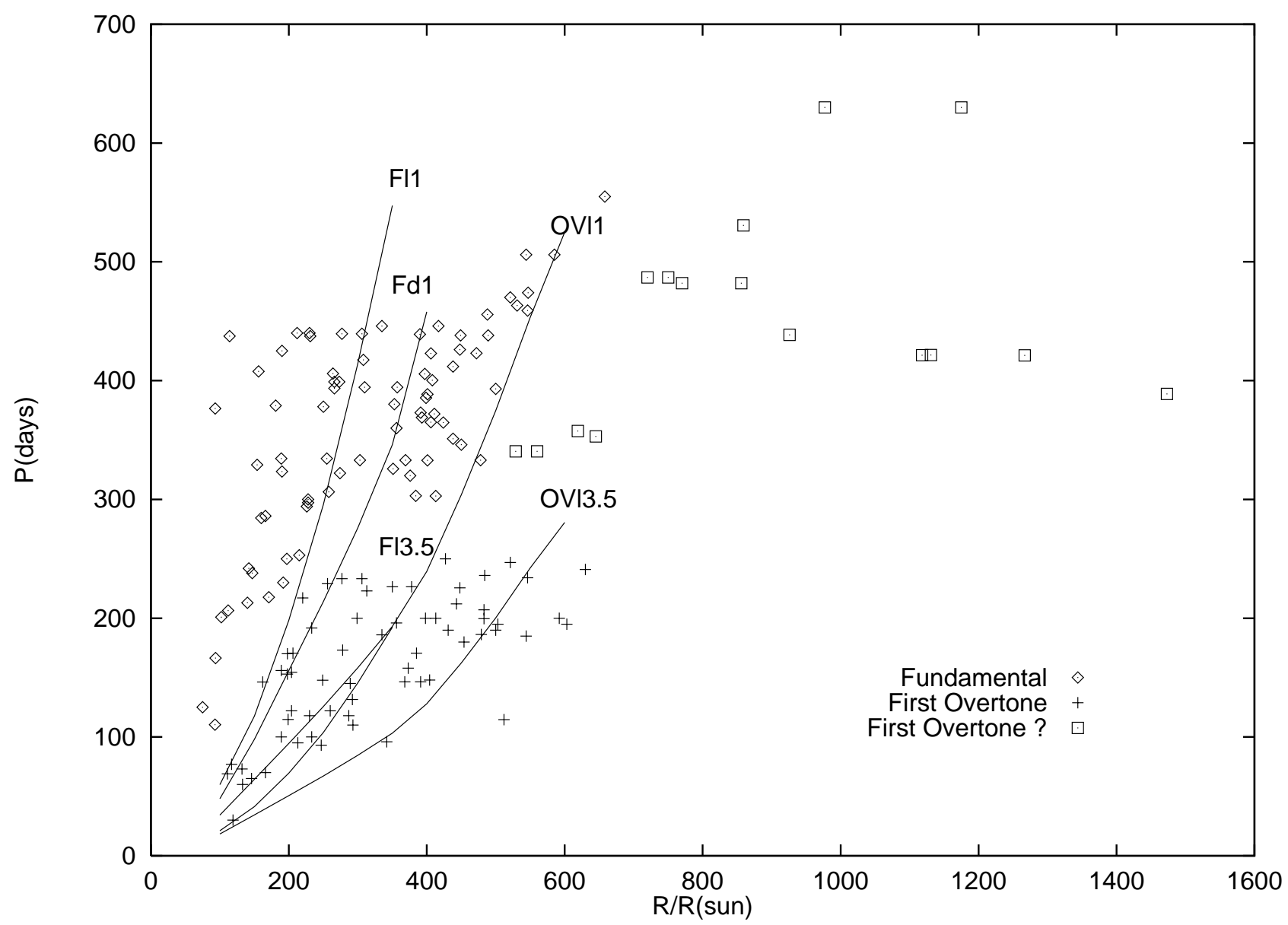

Fig. 1. The period-radius diagram of 155 carbon variables. The theoretical predictions for fundamental (F) and first-overtone (OV) pulsators are shown for masses 1 and $3.5 M_{\odot}$. Linear calculations are labelled with 1 while (non-linear) dynamical ones are referred to as d. The proposed identification of pulsation modes is also shown (see text for details).

are not fully reliable at present, and presented satisfactory fits obtained from linear models.

We have collected the periods available for our pulsating variables, from the GCVS (Kholopov et al. 1985 and further extensions in the literature) and/or from HIPPARCOS (1997) data.

\subsection{The period-radius diagram}

With our goal of mode identification in mind, we established a period-radius diagram of carbon variables. The used photospheric radii were taken from the analysis of Sect. 3. They can be recovered from the absolute bolometric magnitudes and effective temperatures quoted in Table 2 of Paper III at CDS. The accuracy of individual values is rather low. They were essentially intended for statistical purposes. The periodradius (PR) diagram (Fig. 1) is preferred here instead of the period-luminosity plot since, contrary to the latter, model predictions in this diagram are practically independent of the value adopted for the mixing length $\alpha=l / H_{\mathrm{p}}$ of convection (e.g. Figs. 1 and 2 in Ya'ari \& Tuchman 1999). The curves shown in Fig. 1 are those found from linear models of a $1 M_{\odot}$ mass pulsating either in the fundamental mode (F11) or in the firstovertone mode (OV11). Non-linear, i.e. dynamic, results from
Ya'ari \& Tuchman (1996, 1999), fall on the Fd1-curve for the same mass. Applying the proportionalities at constant radius $P \propto M^{-0.84}$ and $P \propto M^{-0.5}$ respectively for fundamental and first-overtone models (Wood et al. 1983; Wood 1990), two curves were established for models of $3.5 M_{\odot}$, which are labelled Fl3.5 and OV13.5 respectively.

It can be seen in Fig. 1 that most carbon LPVs fall in the region of the $1-3.5 M_{\odot}$ theoretical curves, even if both higher and lower masses are indicated for part of the sample. We denote those pulsation masses by $M_{\mathrm{p}}$ hereafter, with $M_{\mathrm{p}} \leq M_{\mathrm{i}}$ due to mass loss. The range of $M_{\mathrm{p}}$ as implied by Fig. 1, is in good agreement with the $M_{\mathrm{i}} \simeq 1-4 M_{\odot}$ range found in Sect. 7.5.2 of Paper III. We emphasize that higher overtones (specially second and third) cannot be excluded, at least for some stars, since calculations suggest they have periods close to those of the first one.

The locus of observations for $R / R_{\odot} \leq 800$ is roughly covered by curves for pulsation masses in the above range. The theoretical curves at constant mass shown in Fig. 1 exhibit curvatures which are oriented upward. The observed data show the opposite trend at small radii, and a flat portion for $R / R_{\odot} \geq 200-250$. In Fig. 1, two main loci are well-correlated with the sampling of Bergeat et al. (1998) in their $M_{\mathrm{K}}-\log P$ diagram: Sample 2 stars are found in the lower part of Fig. 1, 


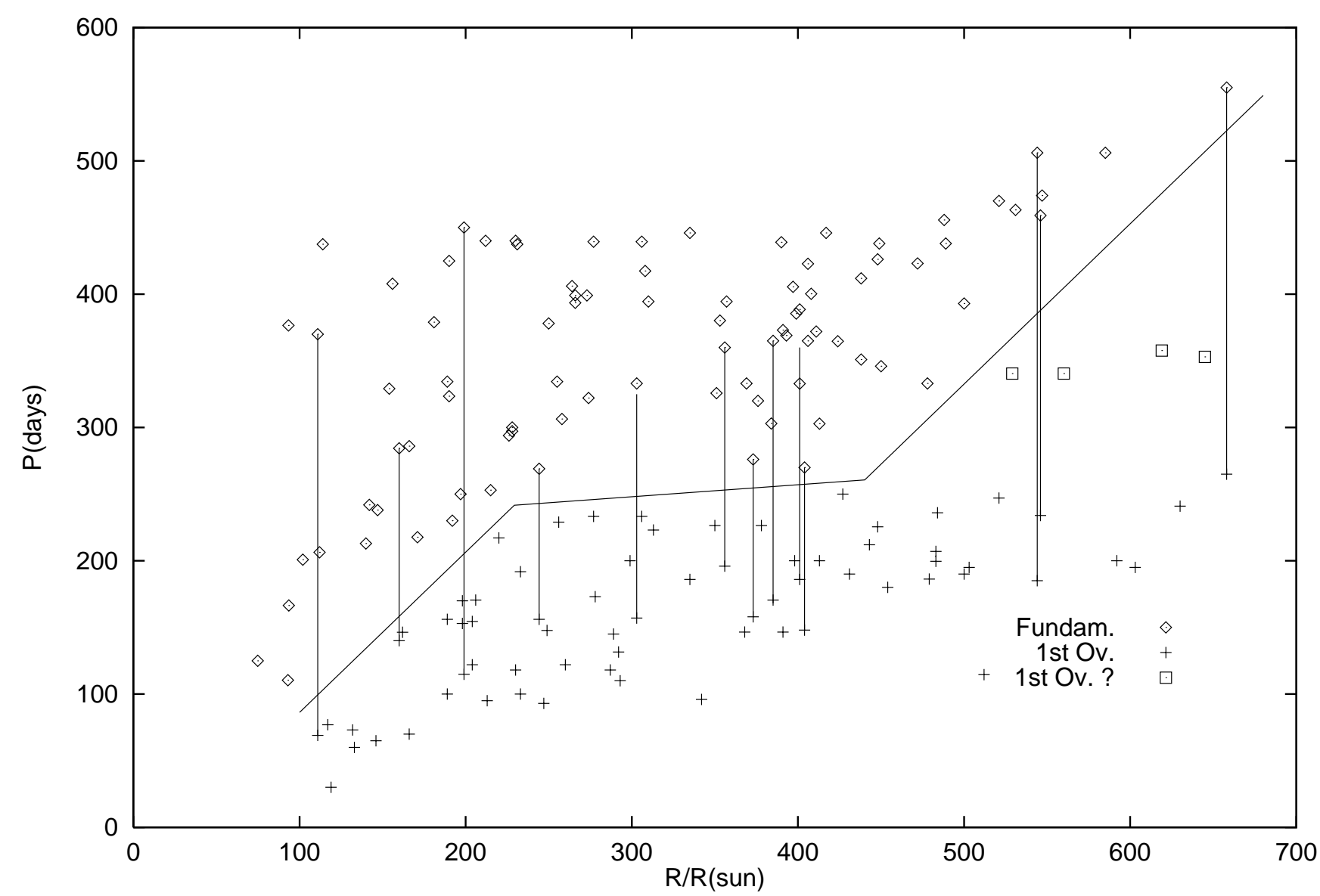

Fig. 2. An enlarged portion of Fig. 1 including 12 carbon variables with confirmed bi-periodicity. The diagram is split into two zones for fundamental and first-overtone pulsators respectively. The adopted separation lines are given by Eqs. (1), (2) and (3). The two nearly horizontal loci over 200-500 $R_{\odot}$ are interpreted in terms of increasing pulsation masses from (0.8 \pm 0.3$) M_{\odot}$ to (3.5 \pm 0.7$) M_{\odot}$ (see text for details).

while Sample 1 stars are located in its upper part. In addition, underluminous giants with long periods (Sample 3) are found on the left side of the Fl1 curve. Both Sample 1 and Sample 2 loci show flat portions. If those samples actually correspond to fundamental and first-overtone pulsators respectively, they should be indicative of a positive correlation of masses with respect to radii, possibly illustrating similar mass-radius relations for both modes. Making use of the evolutionary tracks of Fig. 8 in Paper III, one can find $M_{\mathrm{i}} \propto R^{1.5}$. We shall see in the discussion of Sect. 6 that the exponent appears slightly larger (about $R^{2}$ ) for pulsation masses. Thus, the loci of Fig. 1 are roughly consistent with the hierarchy of evolutionary tracks in the HR diagram. For both modes, the flat parts of period distributions from $200 R_{\odot}$ to $500 R_{\odot}$, can be the result of a $0.6-4 M_{\odot}$ mass distribution. We present in Sects. 2.3 and 2.4, arguments in favor of this interpretation (mode identifications and mass range).

\subsection{Multiperiodicity and mode identification}

A detailed period analysis for 93 red semiregular variables by means of Fourier and wavelet analysis of long-term visual observations was published by Kiss et al. (1999).

A majority of the variables show multiperiodic behavior, typically two or three periods. Kiss et al. concluded that their data can be explained as the direct consequence of different pulsational modes taking place. Their stars are mostly oxygenrich objects. Percy et al. (2001) reported long-term VRI photometry of a sample of small-amplitude red variables, including four carbon stars. Three of them display two periods. We studied this phenomenon in our sample of carbon-rich variables, concentrating here on bi-periodicity. Amongst the 33 possible cases of multiperiodicity we studied, the 12 best-documented cases are shown in Fig. 2 as connected symbols. This figure is an enlarged version of Fig. 1 , restricted to $R / R_{\odot} \leq 700$. With no exception, the longer periods point to the upper "Sample 1" locus while the shorter ones correspond to the lower "Sample 2" locus. These additional data contribute in delineating more confidently the two regions in the diagram. Two segmented lines are shown in Fig. 2 which verify respectively

$P_{1}=1.203\left(R / R_{\odot}\right)-34$

for $R / R_{\odot} \lesssim 230$, and

$P_{1}=0.090\left(R / R_{\odot}\right)+221$

for $R / R_{\odot} \gtrsim 230$. A tentative third half-line is shown for $R / R_{\odot} \gtrsim$ 440 , namely

$P_{1}=1.203\left(R / R_{\odot}\right)-269$ 


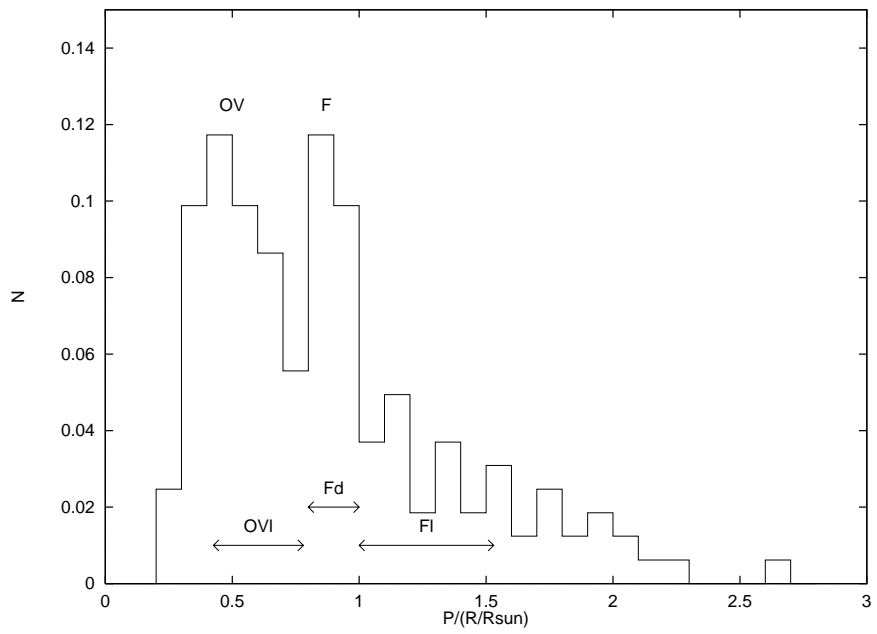

Fig. 3. The distribution of carbon LPVs with respect to the ratio $P /\left(R / R_{\odot}\right)$ of period in days to photospheric radius in solar unit. Two extremas are noticed which correspond to the fundamental $(F)$ and first-overtone $(\mathrm{OV})$ modes, respectively. The ranges found from theoretical predictions $\left(M \simeq 1 M_{\odot}\right)$ of Ya'ari \& Tuchman $(1996,1999)$ are shown with 1 for linear calculations and $d$ for (non-linear) dynamical ones.

assuming an end of distribution at $(3.5 \pm 0.7) M_{\odot}$. For $R / R_{\odot} \gtrsim$ 800 , there is some indication of a new mass increase (4$10 M_{\odot}$ ?) among those rare extreme objects. This is the third part of the distribution which is denoted by "First Overtone?" in Fig. 1, for $R / R_{\odot} \gtrsim 700$.

Identification of modes can then be operated with reasonable confidence. The main problem is probably the possible existence of higher-order overtones (second and third) whose periods are presumably close to those of the first one. The longer periods (Samples 1 and 3) populate the range 270-560 days with a mean $\left\langle P_{0}\right\rangle \simeq 382$ days to be compared to 70-270 days and $\left\langle P_{1}\right\rangle \simeq 166$ days for the shorter periods (Sample 2). We thus obtain a ratio of $2.3 \pm 0.7$ or directly from a larger sample

$\left\langle P_{0} / P_{1}\right\rangle \simeq 2.24 \pm 0.7$

This is close to the mean ratio theoretically calculated $(\geq 2.0)$ for fundamental and first-overtone modes respectively (e.g. Fox $\&$ Wood 1982, their Fig. 3, p. 204). Amongst 10 stars in common with Kiss et al., we found good agreement for 8 carbon variables and discrepancies in two cases $(\mathrm{C} 36=\mathrm{VX}$ And and C2803=U Hya).

\subsection{The distribution in $P / R$}

To confirm the results of Sect. 8.3, we show in Fig. 3, the frequency distribution of the carbon variables with respect to the ratio

$\beta=P /\left(R / R_{\odot}\right)$

that is the slope calculated from origin in the former diagrams. Two maxima are noticed that correspond well to the values of $\beta$ of the OV and F models respectively. Mean masses larger than $1 M_{\odot}$ are however indicated in both cases (see Sect. 8.2).
There are about 10 objects with very large radii $R / R_{\odot} \simeq$ 700-1500. They belong to the CV6 and CV7 groups, and they show evidence for substantial circumstellar shells. We tentatively ascribed pulsation in the first overtone to those stars. The estimated masses in the case of fundamental pulsators would be much too high. Unlikely values $\left(20-30 M_{\odot}\right)$ would be attributed to those extreme objects, in strong disagreement with the theoretical tracks in the HR diagram. Alternatively, their radii could have been overestimated due to undetected circumstellar contributions. This is precisely the sample of extreme stars whose low effective temperatures were derived with some difficulty (Paper I).

\section{The pulsation masses of carbon LPVs}

\subsection{The method}

To estimate pulsation masses, we made use of the PMR relations of Wood (1990) for the fundamental mode and of those of Wood et al. (1983) for the first overtone. Calculated for oxygenrich LPVs, they were already applied by Groenewegen \& de Jong (1994) to LPVs in the LMC, either oxygen-rich or carbonrich. For the fundamental, we thus adopted the period

$$
P_{0}=0.00851\left(R_{\mathrm{p}} / R_{\odot}\right)^{1.94}\left(M / M_{\odot}\right)^{-0.90}
$$

for $M / M_{\odot} \leq 1.5$

and

$P_{0}=0.00363\left(R_{\mathrm{p}} / R_{\odot}\right)^{2.09}\left(M / M_{\odot}\right)^{-0.77}$

for $M / M_{\odot} \geq 2.5$

and an interpolation formula in the intermediate mass-range. Concerning the first overtone, the period is

$P_{1}=Q\left(R_{\mathrm{p}} / R_{\odot}\right)^{1.50}\left(M / M_{\odot}\right)^{-0.50}$

where

$Q=0.038+5.5 \times 10^{-5}\left(P_{1}-100\right)$

for $M / M_{\odot} \leq 0.85$ and $P_{1} \geq 100$

$Q=0.038+4.5 \times 10^{-5}\left(P_{1}-150\right)$

for $0.85 \leq M / M_{\odot} \leq 1.5$ and $P_{1} \geq 150$

$Q=0.038+2.5 \times 10^{-5}\left(P_{1}-300\right)$

for $1.50 \leq M / M_{\odot} \leq 2.5$ and $P_{1} \geq 300$

and $Q=0.038$ in any other case. The accuracy of the radii is not sufficient to allow individual derivation of pulsation masses, and the true parallaxes used were intended essentially for statistical use. The expressions of pulsation masses obtained from (6), (7) and (8) are not proportional to true parallaxes. Averaging individual masses is thus subject to the bias described by Smith \& Eichhorn (1996). A method consists of averaging quantities linear in $\varpi \propto\left(R / R_{\odot}\right)^{-1}$, i.e. $\left(M_{\mathrm{p}} / M_{\odot}\right)^{-\gamma}$ with $\gamma \simeq 0.46,0.37$ and 0.33 respectively for Eqs. (6), (7) and (8). The appropriate version of Eq. (9) has to be selected by trial and error. This is much like averaging inverse radii. Since we are missing information about possible biases on periods, 
Table 1. Mean pulsation masses $\left\langle M / M_{\odot}\right\rangle$ estimated for the photometric groups from Eqs. (6) to (9) and the second method described in Sect. 3.1. Both fundamental $(\mathrm{F})$ and first-overtone $(\mathrm{O})$ modes were used with similar results. First overtone pulsation was assumed for CV6-CV7 stars with very large radii $\left(\mathrm{O}^{\prime}\right)$. Adopted mean masses $\left(M_{\mathrm{ad}}\right)$ are also quoted together with dispersions, for documented groups (both modes): HC5, $\mathrm{CV} 1, \mathrm{CV} 2, \mathrm{CV} 3, \mathrm{CV} 4, \mathrm{CV} 5$, and CV6, illustrating the increase of the average mass along the photometric sequence. Values quoted for HC3, HC4, CV7 and SCV are only indicative. The case of some underluminous CV5-stars is also investigated (see text for details). The spanned range is nearly $0.5-4.2 M_{\odot}$, in good agreement with results of Sect. 7.5.2 in Paper III. Also quoted the mean density referred to the solar value $\rho^{\prime}=10^{8}\langle\rho\rangle /\left\langle\rho_{\odot}\right\rangle$, and the mean surface gravity $g^{\prime}=10^{3}\langle g\rangle$ in SI units, i.e. 10 times its value in CGS units.

\begin{tabular}{|c|c|c|c|c|c|c|c|c|c|c|c|}
\hline G & $\mathrm{m}$ & $\mathrm{n}$ & $\left\langle P_{0,1}\right\rangle$ & $\left\langle\frac{R}{R_{\odot}}\right\rangle_{0,1}$ & $\left\langle\frac{M}{M_{\odot}}\right\rangle$ & $\left\langle M_{\mathrm{bol}}\right\rangle$ & $\left\langle T_{\text {eff }}\right\rangle$ & $\rho^{\prime}$ & $g^{\prime}$ & $\frac{M_{\mathrm{ad}}}{M_{\odot}}$ & Comments \\
\hline $\mathrm{HC} 3$ & $\mathrm{~F}$ & 1 & 125 & 75 & 0.26 : & -3.15 & 3945 & 61: & 13: & 0.3: & $\mathrm{C} 3938=\mathrm{V}$ CrA \\
\hline $\mathrm{HC} 4$ & $\mathrm{~F}$ & 1 & 110 & 93 & $0.47:$ & -3.65 & 3865 & 59: & 15: & 0.5 : & C3319=TT CVn; CH \\
\hline \multirow[t]{2}{*}{$\mathrm{HC} 5$} & $\mathrm{~F}$ & 5 & $290 \pm 70$ & $161 \pm 83$ & 0.53 & -4.18 & 3400 & 13 & 5.6 & & \\
\hline & $\mathrm{O}$ & 2 & $74 \pm 5:$ & $137 \pm 34$ & 0.68 & -3.76 & 3460 & 26: & 10: & $0.6 \pm 0.2$ & \\
\hline \multirow[t]{2}{*}{ CV1 } & $\mathrm{F}$ & 6 & $299 \pm 98$ & $153 \pm 55$ & 0.46 & -3.60 & 3300 & 13 & 5.3 & & \\
\hline & $\mathrm{O}$ & 8 & $99 \pm 42$ & $159 \pm 42$ & 0.59 & -3.76 & 3290 & 15 & 6.4 & $0.55 \pm 0.15$ & \\
\hline \multirow[t]{2}{*}{$\mathrm{CV} 2$} & $\mathrm{~F}$ & 13 & $339 \pm 71$ & $241 \pm 113$ & 1.05 & -4.42 & 3050 & 7.5 & 5.0 & & \\
\hline & $\mathrm{O}$ & 16 & $147 \pm 46$ & $231 \pm 90$ & 0.94 & -4.34 & 3020 & 7.6 & 4.8 & $1.0 \pm 0.2$ & \\
\hline \multirow[t]{2}{*}{ CV3 } & $\mathrm{F}$ & 10 & $355 \pm 73$ & $285 \pm 81$ & 1.52 & -4.58 & 2880 & 6.6 & 5.1 & & \\
\hline & $\mathrm{O}$ & 12 & $159 \pm 54$ & $302 \pm 84$ & 1.62 & -4.73 & 2910 & 5.9 & 4.9 & $1.6 \pm 0.3$ & \\
\hline \multirow[t]{2}{*}{ CV4 } & $\mathrm{F}$ & 14 & $353 \pm 55$ & $299 \pm 129$ & 1.74 & -4.77 & 2770 & 6.5 & 5.4 & & \\
\hline & $\mathrm{O}$ & 8 & $178 \pm 53$ & $358 \pm 157$ & 2.16 & -4.96 & 2780 & 4.7 & 4.6 & $1.9 \pm 0.35$ & \\
\hline \multirow[t]{6}{*}{ CV5 } & $\mathrm{F}$ & 13 & $393 \pm 51$ & $311 \pm 133$ & 1.68 & -4.40 & 2670 & 5.6 & 4.8 & & \\
\hline & $\mathrm{F}$ & 10 & $384 \pm 30$ & $387 \pm 115$ & 3.15 & -4.91 & 2670 & 5.4 & 6.0 & & without 3 underluminous stars \\
\hline & $\mathrm{F}$ & 3 & 423: & 188: & 0.48 & -3.28 & 2645 & 7.3 & 3.7 & 0.5 : & underlum.: RU Pup, T Lyn, RZ Peg \\
\hline & $\mathrm{O}$ & 15 & $201 \pm 26$ & $376 \pm 114$ & 2.14 & -4.76 & 2660 & 4.0 & 4.1 & & \\
\hline & $\mathrm{O}$ & 14 & $200 \pm 24$ & $396 \pm 100$ & 2.25 & -4.87 & 2660 & 3.6 & 3.9 & $2.7 \pm 0.5$ & without 1 underluminous star \\
\hline & $\mathrm{O}$ & 1 & 217: & 220: & 0.45 & -3.61 & 2675 & 4.2 & 2.5 & $0.5:$ & underlum.: AC Pup \\
\hline \multirow[t]{3}{*}{ CV6 } & $\mathrm{F}$ & 13 & $442 \pm 49$ & $479 \pm 110$ & 4.67 & -4.86 & 2460 & 4.3 & 5.6 & & \\
\hline & $\mathrm{O}$ & 4 & $212 \pm 23$ & $422 \pm 119$ & 2.78 & -4.58 & 2465 & 3.7 & 4.3 & & \\
\hline & $\mathrm{O}^{\prime}$ & 7 & $458 \pm 67$ & $779 \pm 97$ & 3.97 & -5.88 & 2250 & 0.84 & 1.8 & $4.2 \pm 0.8$ & $\mathrm{O}^{\prime}=$ supposed 1 st overtone \\
\hline CV7 & $\mathrm{O}^{\prime}$ & 7 & $444 \pm 107$ & $1040 \pm 370$ & 8.24 & -5.72 & 1970 & 0.73 & 2.1 & 8.2: & $\mathrm{O}^{\prime}=$ supposed 1st overtone \\
\hline \multirow[t]{2}{*}{ SCV } & $\mathrm{F}$ & 1 & 365: & 406: & 3.83: & -5.49 & 2880 & 5.7: & 6.4: & & V346 Aur \\
\hline & $\mathrm{O}^{\prime}$ & 3 & 227: & 533: & 5.04: & -5.83 & 2995 & 3.3: & 4.9: & 4.7: & $\mathrm{O}^{\prime}=$ supposed 1 st overtone \\
\hline
\end{tabular}

we suppose that their influence can be neglected here. A second method is to consider a fictitious star with inverse radius equal to the average inverse radius of the studied sample and period equal to the average period. Those quantities are then introduced in Eqs. (6) to (9). Differences between both methods are most often small and never exceed $15 \%$. We finally adopted the second method, which we consider as slightly more favorable. If the distribution for a given sample was strongly nonGaussian, a bias toward lower masses might intervene. This is not the case for the HC- and CV-samples studied in Sect. 3.2.

\subsection{The results}

The available data is practically limited to the HC5, SCV and CV1-CV7 photometric groups, some of them being poorly documented. The adopted period (either fundamental mode or first overtone) is the mean of individual values in the sample. The mean radius was obtained from the values of the $C_{\mathrm{R}}$-coefficient as described in Sects. 3.1 and 4 of Paper III. The results were given in Table 1 of Paper III. The mean pulsation mass increases along the sequence of groups from $\simeq 0.6 M_{\odot}$ at HC5$C V 1$ to $\simeq 4 M_{\odot}$ at $C V 6-C V 7$.

The mean pulsation periods are quoted for each mode, identified as described in Sect. 2. The adopted mode is doubtful for a few percent of the data that might prove to be misclassified later on, but the mean values should not be seriously affected. The mean values of photospheric radii are given for variables pulsating in either mode. They can differ from values quoted in Table 3 of Paper III which includes all the carbon giants of our initial sample, even if they are not LPVs (e.g. even for irregular variables). It was shown in Table 4 of Paper III that, on average, the SR- and Mira-variables are slightly brighter than the Lb-ones. We have also operated a detailed comparison, group per group, with the conclusion: there is no significant difference in terms of $M_{\text {bol }}$ between the irregular Lb-stars and the whole sample. The difference noted in Table 4 of Paper III is actually an artifact of the concentration of irregular variables in earlier (thus less luminous) photometric groups (say HC5 to CV3) than SRs and Miras (see Fig. 8 of Paper II). 
Small mean pulsation masses are found in the groups HC5 and CV1. One is tempted to question the applicability of the PMR relations as written in Sect. 3.1. They were established for oxygen-rich variables, and the effect of opacities specific for carbon-rich atmospheres on such models remains to be investigated. We however note that

- the values $0.5-0.6 M_{\odot}$ are physically possible, allowing only tiny envelopes depleted by mass loss,

- the (0.6 \pm 0.2$) M_{\odot}$-mean of HC5-stars is compatible with what is expected from $\mathrm{CH}$ stars among HC-stars (represented in $\mathrm{HC}$, e.g. $\mathrm{C} 327=\mathrm{V}$ Ari). It has been argued that they all are members of double stars (McClure \& Woodsworth 1990) whose companion is a white dwarf of nearly $0.6 M_{\odot}$. The statistical study of 8 systems yielded $\langle M\rangle \simeq(0.8 \pm 0.1) M_{\odot}$.

Incidentally, we obtained the same value $\left(0.6 M_{\odot}\right)$ for the RCBvariable $\mathrm{C} 4098=\mathrm{V}$ CrA (F9g) whose period is 75 days. The other end of the distribution $\left(4 M_{\odot}\right)$ is not well-established. The existence of a few massive CV6-CV7 ( $\left.\gtrsim 4 M_{\odot}\right)$, with strong mass loss and optically thick circumstellar shells, seems indicated on the grounds of the available data. The HIPPARCOS sample contains unfortunately only Miras of intermediate optical thickness like R Lep. Some SCV-stars intermediate between the bright (intrinsic) $\mathrm{S}$ stars and the $\mathrm{CV}$-stars may also be more massive than the above $4 M_{\odot}$ upper limit.

An interesting question comes from the underluminous carbon giants already found when studying the period-luminosity diagrams of the present Galactic carbon stars (Sample 3 of Bergeat et al. 1998), and those of their analogues in the LMC. Those CV5-CV6 stars are found at $\left\langle M_{\mathrm{bol}}\right\rangle \simeq-3.3$ that is about 1.4 mag below the locus of standard CV5-stars (say $\left.\left\langle M_{\mathrm{bol}}\right\rangle \simeq-4.7\right)$. Samples without underluminous stars have been also considered, resulting in increased estimates of mean masses. The mean mass deduced for 4 underluminous CV5stars, namely RU Pup, T Lyn, RZ Peg and AC Pup, amounts to $(0.5 \pm 0.15) M_{\odot}$, a value again compatible with a practically stripped core. Such underluminous giants are also observed in the LMC (Bergeat et al. 1998). Spurious results from HIPPARCOS may accidentally contribute but their existence seems beyond doubt. The above mean mass we found suggests that they experienced strong mass loss, evolving from standard CV-stars. We note that their mean masses, luminosities and radii are similar to those of the CV1- or HC5-group, the main difference lying in mean effective temperatures about 650 to $800 \mathrm{~K}$ higher in the latter groups. More speculatively, they could be low-luminosity "interpulse" objects or post-TP AGB stars evolving toward HC5-CV1 at nearly constant luminosity. Such a possibility is suggested by theoretical tracks (e.g. Lattanzio 1987; Sackman et al. 1993). Many RCB variables and $\mathrm{HdC}$ stars with similar low masses are considered as bornagain objects (Sect. 7.2 of Paper III). The results in Table 1 can be summarized as follows; (1) a marked increase of mean masses along the HC5-CV7 sequence, to be discussed later, (2) the absence of large systematic differences between mean values deduced from both modes, (3) the consistency of extreme mean values $\left(0.5-4 M_{\odot}\right)$ with prediction from evolutionary tracks and mass loss operating, (4) the mean stellar density steadily decreases along the sequence as expected, and (5) the mean surface gravity is practically constant, close to $0.005 \mathrm{SI}$ that is 0.5 CGS $(\log g \simeq-0.3)$.

\section{The mass-luminosity diagram}

The values of Table 1 are mean values obtained over an unknown mass distribution. Intrinsic dispersion and errors from parallaxes both contribute. The main feature is the increase of mean masses along the sequence of photometric groups, at least from HC5 ( $T_{\text {eff }} \simeq 3500 \mathrm{~K}$ ) to CV7 (about $T_{\text {eff }} \simeq 2000 \mathrm{~K}$ ). This is also a sequence of increasing luminosities from about $1700 L_{\odot}$ to $17000 L_{\odot}$ (see Table 3 of Paper III). It was shown in Paper I that the mean $\mathrm{C} / \mathrm{O}$ ratio and its dispersion increase along this sequence (at least from CV2 to CV6). The SEDs are obviously affected by increasing opacities (gas + grains) along the same sequence. This is also a sequence of increasing mass loss and increasing CS dust emission of $\mathrm{SiC}$ at $11 \mu \mathrm{m}$ (Bergeat 2002c).

Our mean masses in Table 1 are shown in Fig. 4, which is a mass-luminosity diagram, making use of the absolute bolometric magnitudes. Mean luminosities increase with mean pulsation masses as predictable from initial masses of theoretical tracks. It was expected since mean pulsation mass increases along the sequence of groups (Table 1), which is a sequence of increasing mean luminosities (Table 3 of Paper III). The locations of the onset and tip of TP-AGB for $Z=0.008$ are adapted from Fig. 6 of Marigo et al. (1999). A good agreement is observed between our results and those of predictions. Typical dispersion bars are shown as double arrows. Taking them into account, it can be seen that most of the results are close to the tip (solid line) of the TP-AGB region. It is also likely that, on average, the metallicity is somewhat larger than $Z=0.008$ and less than $Z=0.02$ (solar value). The tip-line shifts slightly downward in Fig. 4 for such a value (say $Z=0.015$ ). This conclusion concerning location with respect to TP-AGB tip and the above-mentioned sequence of increasing mean luminosity vs. mean pulsation mass, suggests that, on average, the carbon giants are located close to their track end in the HR diagram. In addition, this end shifts toward lower effective temperatures for increasing masses, i.e. increasing $\mathrm{C} / \mathrm{O}$ ratios and opacities (Paper I). This trend is seen for $Z=0.02$ in Fig. 8 of Paper III, and when comparing the track for $M=1 M_{\odot}$ to that for $M=4 M_{\odot}$ (but not clearly for $M=2 M_{\odot}$ ). It is worth noting that tracks ending at too high effective temperatures may result from opacities missing in the models.

Considering $l=\left\langle L / L_{\odot}\right\rangle$ and $m=\left\langle M / M_{\odot}\right\rangle$, we found that the unweighted data satisfy the mean relation

$\log l \simeq(0.72 \pm 0.07) \log m+(3.58 \pm 0.12)$

with a correlation coefficient of $\rho^{2} \simeq 0.87$. Restricting the fit to the best-documented results from CV2 to CV5 (over 97 stars, eight mean masses averaged in the $1-3 M_{\odot}$ range), we obtained $\log l \simeq(0.497 \pm 0.075) \log m+(3.658 \pm 0.034)$

with $\rho^{2} \simeq 0.88$. The intermediate part of the mass-luminosity relation is thus very nearly

$l \propto m^{1 / 2}$

a result we comment on in Sect. 6. 


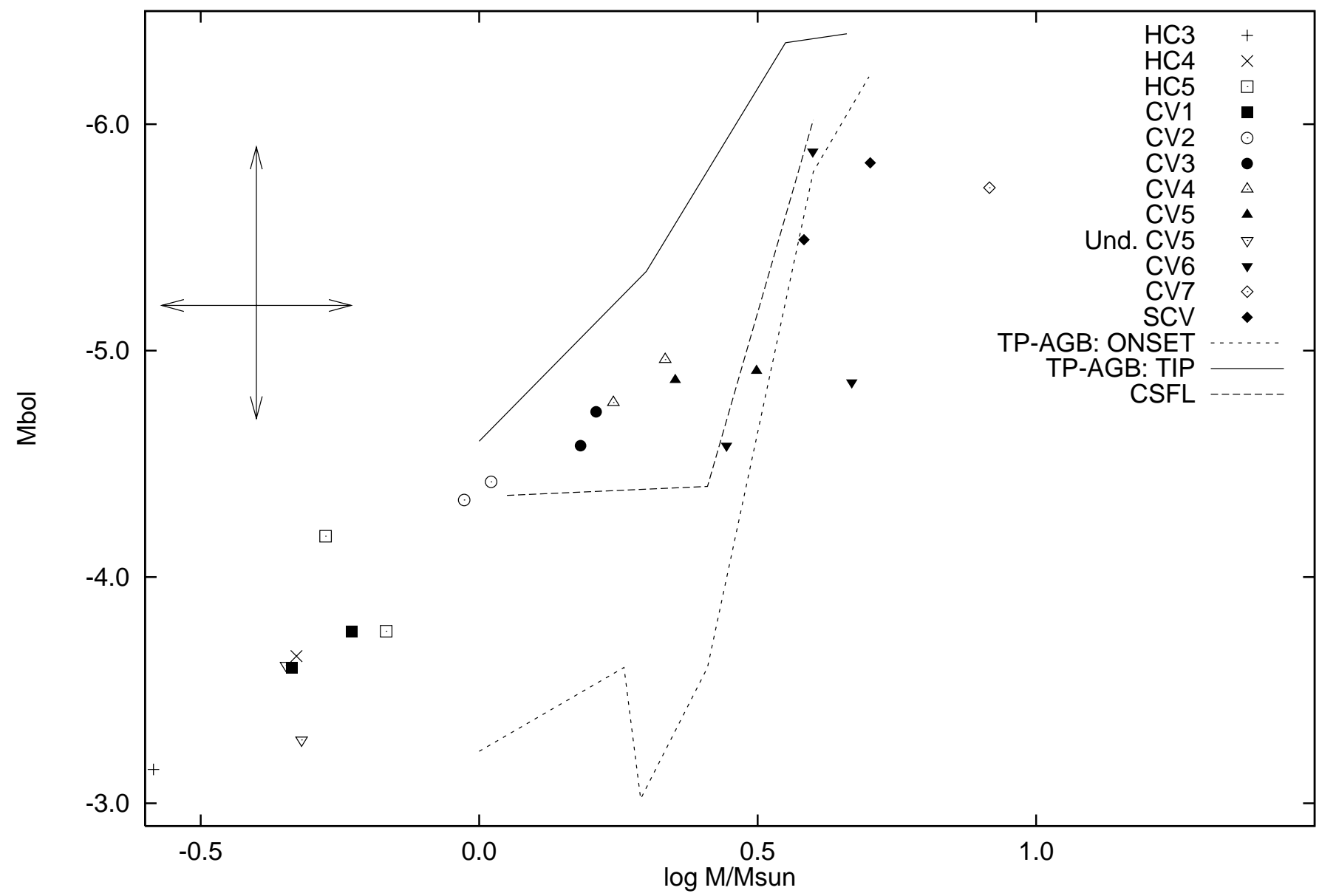

Fig. 4. A diagram of mean absolute bolometric magnitudes vs. mean pulsation masses in solar unit on a log scale (Table 1). For the sake of clarity, only typical dispersions in both coordinates, are shown as arrows. The TP-AGB zone is delineated by theoretical lines (onset and tip; $Z=0.008)$ and a carbon star formation line (CSFL) is shown (see text for details).

There is also a reasonable correspondence with the carbon star formation line (CSFL) for $Z=0.008$ from Marigo et al., specially that of their intermediate case $\mathrm{B}\left(\log T_{\mathrm{b}}^{\mathrm{dred}}=6.4\right.$ and $\lambda=0.5$; dashed line in Fig. 4). The observed stars actually span a large range in metallicities.

Estimates of mean stellar densities are given in Table 1 (Col. 9). These data can be compared to the result of internal structure calculations. It is seen that they continuously decrease from HC5 to CV7 (a factor of about 22). In addition, estimates of surface accelerations or gravities are quoted in Table 1 (Col. 10). These data, useful for atmosphere modeling, complement the effective temperatures provided in Paper I. The values are around $g=(5.1 \pm 0.7) 10^{-3}$ SI or $\simeq 0.5$ CGS $(\log g \simeq-0.3)$. They only diminish by a factor of 2 at CV6-CV7, for the extreme objects with the overtone assumption $\left(\mathrm{O}^{\prime}\right)$, but this result is only indicative.

In summary, it appears that the $C V$-giants are close to the tip of the TP-AGB region, and close to the end of their track towards the right in the HR diagram. With larger masses, this end shifts toward lower effective temperatures and higher luminosities.

\section{C/O ratio vs. effective temperature for the three populations of carbon-rich giants}

The existence of at least three populations of Galactic carbon giants was demonstrated in Paper II on the grounds of vertical space distribution and kinematics. Most HC-stars are members of the thick disk, and their sample is contaminated by a spheroidal component, namely the HC-stars classified as $\mathrm{CH}$ stars by spectral criteria (see also Hartwick \& Cowley 1985). Most CV-stars are members of the old (thin) disk. We have found this result again in Sect. 6 of Paper III, where the former two populations correspond to the maximum in the LF at $M_{\text {bol }} \simeq-1.75$ or -1.25 (see Fig. 5 of Paper III) and the latter one can be associated with the second maximum at $M_{\mathrm{bol}} \simeq$ -4.75 . With a separation of more than three magnitudes, the maxima are clearly disentangled. The transition in the HR diagram between the former two populations (HC-stars) and the latter one (CV-stars) is shown in Fig. 9 of Paper III, and nearly coincides with the CSFL for TP-AGB stars having experienced TDU. When compared with the theoretical evolutionary tracks, the former locus (HC-stars) appears shifted leftward if compared to the latter one (CV-stars). We interpret this shift in terms of lower metallicity on average, in the former lowmass old stars (see references cited hereafter in this section), as 


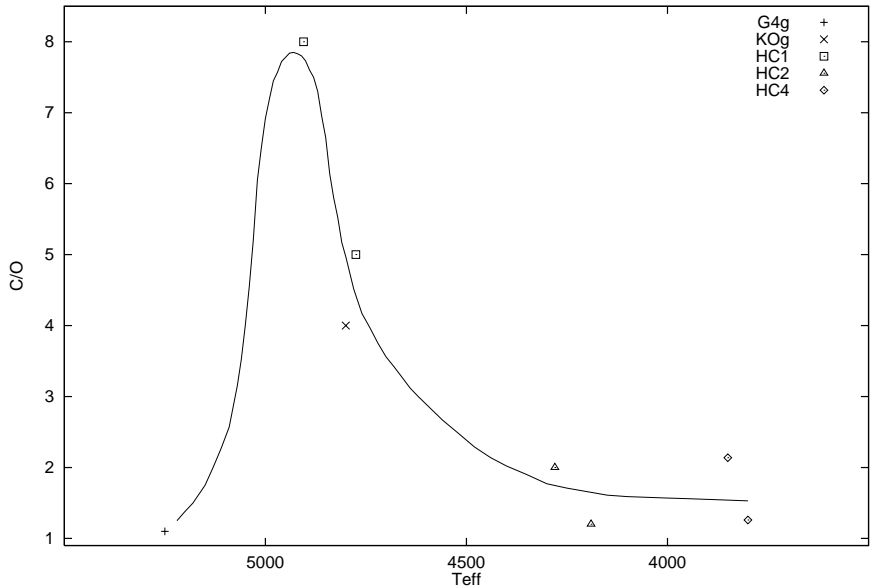

Fig. 5. The $\mathrm{C} / \mathrm{O}$ abundance ratio as a function of effective temperature for the $\mathrm{CH}$ stars (classified in an oxygen-rich or an HC-group). A curve was drawn by hand through the eight available points, a maximum being apparent near $4900 \mathrm{~K}$.

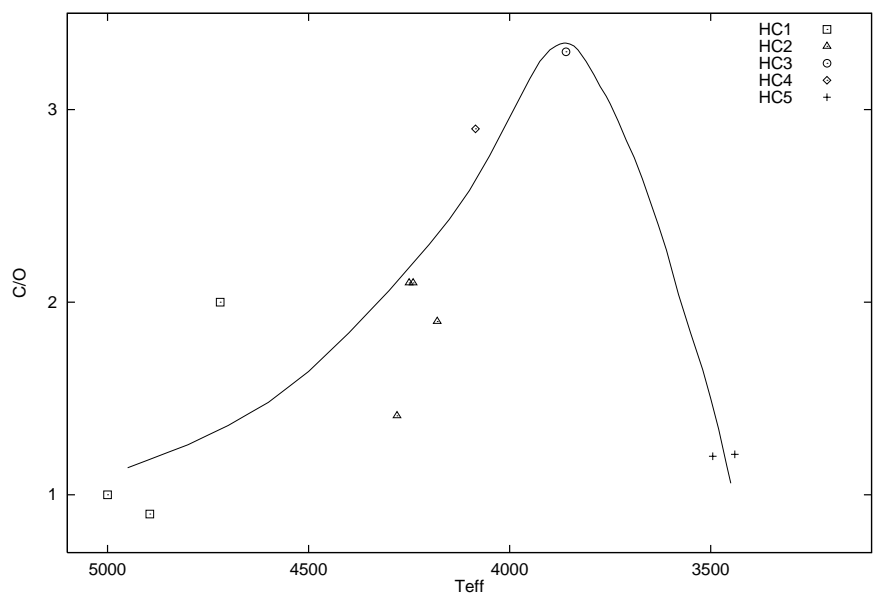

Fig. 6. The same diagram as Fig. 5 but for the HC (Hot Carbon) stars not classified $\mathrm{CH}$ stars on spectroscopic grounds. A curve was drawn by hand through the eleven points available, a maximum of the $\mathrm{C} / \mathrm{O}$ ratio being apparent near $3870 \mathrm{~K}$.

suggested by the comparisons of tracks for $Z=0.008$ to those for $Z=0.02$.

We display now further evidence in favor of the three above-mentioned populations. This is the carbon to oxygen $(\mathrm{C} / \mathrm{O})$ abundance ratio shown as a function of effective temperature in Figs. 5, 6 and 7 respectively for the $\mathrm{CH}$ stars, the $\mathrm{HC}$ stars not classified as $\mathrm{CH}$ stars, and finally the CV-stars. The effective temperatures, essentially from Paper I, can be found in Table 2 of Paper III, at CDS. The C/O ratios are taken from Dominy (1984), Lambert et al. (1986), Vanture (1987), Kipper \& J $\phi$ rgensen (1994), Abia \& Isern (1996), and Kipper et al. (1996). We concentrated on values derived from comparisons of observed spectra to model atmospheres, and ignored values found in the literature when deduced from the calibration of a color index from photometry. Only $\mathrm{CH}$ stars identified from detailed spectral analysis were kept, excluding the objects mentioned as "CH-like" or "CH-candidates".

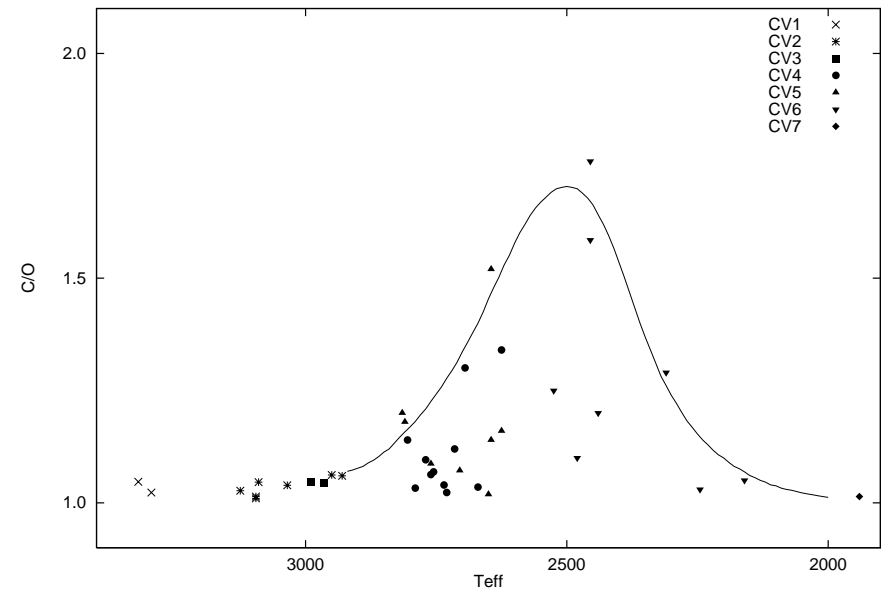

Fig. 7. The same diagram as Fig. 5 for the CV (Carbon Variables) stars which are the coolest ones. There are 39 stars available populating an area below the curve plotted by hand. This "curve of maximas" shows a maximum near $2500 \mathrm{~K}$.

The most remarkable feature in Figs. 5, 6 and 7 is the existence of maxima whose positions and amplitudes are

- $(\mathrm{C} / \mathrm{O}) \max \simeq 8$ at $\left(T_{\text {eff }}\right) \max \simeq(4900 \pm 100) \mathrm{K}$ for the $\mathrm{CH}$ stars,

$-(\mathrm{C} / \mathrm{O}) \max \simeq 3.3$ at $\left(T_{\text {eff }}\right) \max \simeq(3870 \pm 100) \mathrm{K}$ for the $\mathrm{HC}$-stars not classified as $\mathrm{CH}$ stars,

- and $(\mathrm{C} / \mathrm{O}) \max \simeq 1.5$ to 1.75 , at $\left(T_{\text {eff }}\right) \max \simeq$ $(2500 \pm 100) \mathrm{K}$ for the $\mathrm{CV}$-stars, but with most values close to unity.

Thus, the maximum of the $\mathrm{C} / \mathrm{O}$ ratio shifts toward lower effective temperatures from one population to the next and its amplitude decreases correspondingly. It should be noted that in Figs. 5 and 6 documented with only eight and eleven stars respectively, it is not clear whether the tentative curves shown are truly mean curves or only upper limits for the loci. In Fig. 7, there are $39 \mathrm{CV}$-stars and the case is clear. It is possible to estimate an approximate maximum $\mathrm{C} / \mathrm{O}$ value for every effective temperature, resulting in the curve shown. The result of Paper I is thus confirmed (increasing mean values and dispersions of the $\mathrm{C} / \mathrm{O}$ ratio along the sequence of the photometric $\mathrm{CV}$-groups until CV6). Making use of effective temperatures instead of photometric groups (and of an enlarged sample), however, reveals a decrease of photospheric $\mathrm{C} / \mathrm{O}$ for $T_{\text {eff }} \lesssim 2500 \mathrm{~K}$. This is precisely the temperature below which circumstellar dust (carbon and $\mathrm{SiC}$ ) efficiently condenses, as shown by infrared excesses of circumstellar origin. The model atmospheres used in the above-mentioned photospheric studies did not include dust species. The maximum may thus be apparent in Fig. 7, referring to gas $\mathrm{C} / \mathrm{O}$ only. We have not included here the very evolved object $\mathrm{C} 2619=\mathrm{CW}$ Leo $=\mathrm{IRC}+10216\left(\mathrm{CV} 7,\left\langle T_{\mathrm{eff}}\right\rangle \simeq 2000 \mathrm{~K}\right)$ whose $\mathrm{C} / \mathrm{O}$ ratio is estimated to be $1.4-1.5$. Due to large nonspherical obscuration (e.g. Knapik et al. 1999 and references therein) this is a circumstellar value obtained well above the region of dust condensation with large $\mathrm{C}$-depletion in the gas. Thus this is not in contradiction with our results in Fig. 7, that correspond to nearly photospheric values. 
As expected, low-mass HC-stars $\left(M_{\mathrm{i}} \leq 1.15 M_{\odot}\right)$ with low metallicities do exhibit on average higher $\mathrm{C} / \mathrm{O}$ ratios than the $\mathrm{CV}$-stars with higher masses and metallicities close to solar value. The main results and general trend are consistent with evolutionary tracks shifted leftward (increasing effective temperatures) in the HR diagram, with decreasing metallicity. It is usually assumed that $\mathrm{CH}$ stars are binary members whose carbon enrichment occurred through mass transfer from a former TP-AGB primary, now a white dwarf (McClure 1989; McClure \& Woodsworth 1990). No evidence for binarity was found in the HC-stars not classified as $\mathrm{CH}$ stars, and their status is not clear at all (Sect. 7 of Paper III). The CV-stars are clearly identified with stars enriched in carbon through TDU in TP-AGB objects (Sect. 7 of Paper III). We can summarize those facts by the comments "presumably extrinsic", "unknown" and "intrinsic" for the three categories respectively. The high $\mathrm{C} / \mathrm{O}$ ratios observed in most $\mathrm{CH}$ stars are however somewhat surprising for stars that are usually considered as the equivalent of BaII stars ("extrinsic" objects) in the spheroidal component.

\section{Summary and discussion}

Identification of pulsation modes (fundamental, overtone, presumably the first) of carbon LPVs was provided in a periodradius diagram. The comparison with theoretical tracks in Figs. 1 and 3 and a study of stars with bi-periodicity (Fig. 2), were of considerable help. Mean pulsation masses were derived from theoretical PMR relations. They are quoted in Table 1 together with mean densities and mean surface gravities. The range of pulsation masses was found to be $0.6-4.0 M_{\odot}$ for the majority of carbon-rich giants. This is in good agreement with the previous studies made on various subsamples by Claussen et al. (1987), Thronson et al. (1987), Zuckerman \& Dyck (1989), and Barnbaum et al. (1991). The agreement is also good with studies on individual sources like IRC +10216 , from the comparison of theoretical nucleosynthesis models and measurements of abundances in circumstellar envelopes (specifically LMS-models favored and a $5 M_{\odot}$-model rejected from the isotopic ratios observed in this object: Kahane et al. 2000). A mass-luminosity diagram was derived (Fig. 4) and discussed. It should be kept in mind that it applies to mean values of both quantities, with large standard deviations on both of them. Intrinsic ranges are involved since parameters like abundances span large domains. Finally, the diagrams of $\mathrm{C} / \mathrm{O}$ abundance ratios vs. effective temperatures were constructed (Figs. 5, 6 and 7) for the three classes of carbon-rich giants in our sample ( $\mathrm{CH}$ stars, $\mathrm{HC}$ not classified $\mathrm{CH}$, and $\mathrm{CV}$ ).

Having summarized the main results of the present paper, we discuss them in a wider perspective. The comparison of various LFs in Figs. 5, 6 and 7 of Paper III, and the study of the variations of the $\mathrm{C} / \mathrm{O}$ abundance ratio vs. effective temperature (Figs. 5, 6 and 7), confirmed the existence of three samples of carbon-rich giants in the Sun's vicinity, as shown in Paper II, on the grounds of space distributions and kinematics

- the CH stars (from spectroscopic criteria), classified in either HC-groups or oxygen-rich groups (members of the spheroidal component; $(\mathrm{C} / \mathrm{O}) \max \simeq 8$ at $\left(T_{\mathrm{eff}}\right) \max \simeq$ $4900 \mathrm{~K}$ ),

- the HC-stars not classified CH stars (mainly members of the thick Galactic disk, with a LF similar to that of the MWB; $(\mathrm{C} / \mathrm{O}) \max \simeq 3.3$ at $\left.\left(T_{\text {eff }}\right) \max \simeq 3870 \mathrm{~K}\right)$,

- the CV-stars (mainly members of the old thin disk, with a LF similar to that of the $\mathrm{LMC}((\mathrm{C} / \mathrm{O}) \max \simeq 1.5-1.75$ at $\left.\left(T_{\text {eff }}\right) \max \simeq 2500 \mathrm{~K}\right)$.

The metal-poor systems, like the SMC $(Z=0.004)$ and spheroidal dwarf galaxies, display faint carbon stars such as the Galactic HC-stars. The two maxima of the SMC-LF are however shifted from the Galactic and LMC ones (see Figs. 5, 6 and 7 of Paper III). This probably corresponds to evolutionary tracks shifted toward larger luminosities and higher effective temperatures, for decreasing $(Z)$ metallicity. The $43 \mathrm{CH}$ stars in the outer LMC halo (Hartwick \& Cowley 1988) are much brighter (Feast \& Whitelock 1992; $-4 \gtrsim M_{\text {bol }} \gtrsim-6$ ) than the Galactic ones.

Prochaska et al. (2000) found that the thick disk stars had a chemical enrichment history similar to the metal-rich halo stars $([\mathrm{Fe} / \mathrm{H}] \simeq-1)$. They also concluded that the thick disk abundance patterns are in excellent agreement with the chemical abundances observed in the metal-poor bulge stars, suggesting the two populations formed from the same gas reservoir at a common epoch.

It was shown that, globally, the mean photospheric radius and mean luminosity increases with the mean effective temperature, along the sequence of the photometric groups (HC and $\mathrm{CV}$, with the exception of the hottest one $\mathrm{HCO}$ which is brighter than $\mathrm{HC} 1$, and oxygen-rich groups are still brighter at even higher temperatures). The results are quoted in Table 3 of Paper III and the corrections for the Malmquist bias, although small, were given as well. The mean radii and luminosities increase along the sequence "Constant, Lb, SRb, SRa and Miras" of increasing variability, while mean effective temperature decreases. It corresponds to stars increasingly later on average, along the photometric sequence (see Fig. 8 in Paper II).

In the theoretical HR diagram, a majority of carbon-rich stars are found between the evolutionary tracks of initial masses $M=0.8 M_{\odot}$ and $M=4 M_{\odot}$ for $Z=0.02$. The loci may also be fit with lower masses tracks, when lower metallicities are considered. The derived pulsation masses $\left(0.5-4 M_{\odot}\right)$ are in good agreement with those initial masses. On a small sample, Alksnis et al. (1998) found a reasonable agreement with tracks of (0.7-4 $\left.M_{\odot}\right)$, making use of some observed parallaxes. For S stars, Van Eck et al. (1998) selected 1.5 to $5 M_{\odot}$ model tracks. The loci of $\mathrm{C}$ and $\mathrm{S}$ stars overlap in the HR diagram. The initial $\mathrm{O} / \mathrm{H}$ ratio and mixing history determine whether a star of given mass and luminosity is now a $\mathrm{C}$ star or a $\mathrm{S}$ star. The Ba II giants $\left(\left\langle M_{\mathrm{bol}}\right\rangle \simeq-0.3 \pm 1.3\right)$ are found fainter, on average, than the early $\mathrm{HC}$-stars, and coincide with the clump observed in many clusters. Most RCB variables and HdC stars range from $M_{\text {bol }} \simeq-1$ to -4 against -0.2 to -2.4 for those of the three population II Cepheids in the sample (mean pulsation mass $\simeq 0.6 M_{\odot}$ as expected).

The CV-stars are located at the ends of their TP-AGB tracks and those ends are located at increasingly higher luminosities 
(tip of TP-AGB), shifting toward lower effective temperatures due to increasing opacities occurring in the atmospheres. Increasingly larger $\mathrm{C} / \mathrm{O}$ ratios responsible for that are actually observed (see Fig. 16 in Paper I and Fig. 7 in the present paper). The apparent decrease at $T_{\text {eff }} \leq 2500 \mathrm{~K}$ is likely the effect of carbon atoms trapped in increasing numbers of $\mathrm{SiC}$ and carbon grains.

The observed general trend of increasing luminosities and radii along the $\mathrm{HC}-\mathrm{CV}$ photometric sequence for decreasing mean effective temperatures is the consequence of stellar evolution along the RGB and then the AGB. Some peculiar objects, including hot oxygen-rich stars (RCB variables, HdC stars, carbon cepheids), were discussed and most objects previously classified in luminosity classes II or even I on spectroscopic grounds proved to be fainter than true supergiants or bright giants. The HC-giants were found to be, on average, brighter than the BaII giants whose main concentration coincides with the clump. A second version of the HR diagram (Fig. 9 in Paper III) was restricted to the carbon giants. The quasi-vertical boundary line between the HC-region (left) and the CV-region (right) is nearly identical to the Carbon Star Formation Line (CSFL; e.g. Scalo 1976) associated with the third dredge-up in stars of various masses reaching the TP-AGB phase, for a given metallicity (Iben \& Renzini 1983; Busso et al. 1999; Marigo et al. 1999). The leftward evolution of this CSFL with decreasing metallicity is illustrated in Fig. 4 of Westerlund et al. (1995). The lower limit to the transition in luminosities intervenes at $M_{\mathrm{bol}} \simeq-3.6 \pm 0.4$ which is in agreement with $M_{\text {bol }} \leq-4.0 \pm 0.4$ obtained by Marigo et al. (1999) from evolutionary calculations. The positions of Tc-stars were also shown with a barycenter at $M_{\text {bol }} \simeq-4.6(-3.8 ;-5.8)$, about 1 mag brighter than the above-mentioned lower limit for TPAGB carbon stars. The $C V$-giants and part of the HC5-objects, with $0.5-4 M_{\odot}$ and aged 0.2 to 10-12 Gyr, members of the thin disk, can be identified with TP-AGB stars experiencing TDU. The situation is far less clear for the (thick disk members) HCstars. Various models may be considered for those very old (11 Gyr?), low mass stars (initial value $\lesssim 1.1 M_{\odot}$, present one $\simeq 0.5-0.8 M_{\odot}$ due to mass loss).

With the exception of $\mathrm{CH}$ stars, no evidence for binarity was found in any of those stars (McClure 1997a), and coalescence of components in a former binary system was invoked. It seems unlikely on the basis of low pulsation masses $\left(0.5 M_{\odot}\right)$ found for $\mathrm{HC} 3$ to HC5-stars, and the high frequency of those objects (at least $24 \%$ of carbon stars, including $6 \%$ for $\mathrm{CH}$ stars: Paper II). The "extrinsic" models with mass transfer in a binary system, on a dwarf or giant, should not be considered any longer for $\mathrm{HC}$-stars that are not $\mathrm{CH}$ stars. We are left with the following possibilities

- (1) unpredicted extra mixing on RGB or E-AGB (in a low mass star or around a almost stripped-off core), or sufficient dredge-up of carbon occurring at He-flash, as proposed by Westerlund et al (1991a) for the faint MWB carbon stars and Westerlund et al. (1995) for the faint SMC carbon giants,

- (2) low-mass stars near minimum in an interpulse phase or post last pulse (e.g. $1 M_{\odot}$ models of Sackman et al. 1993),
- (3) RGB or E-AGB carbon giants evolved from dwarfs born with $\mathrm{C} / \mathrm{H} \gtrsim \mathrm{O} / \mathrm{H}$ at low $\mathrm{O} / \mathrm{H}$ (no carbon dragged up needed); in extremely metal-poor stars, Norris et al. (2001) confirmed large supersolar values of $[\mathrm{C} / \mathrm{Fe}]$ that can hardly be attributed to internal mixing effects.

Some extra mixing like cool bottom processing (CBP) is needed to explain the isotopic abundance ratios (specially ${ }^{12} \mathrm{C} /{ }^{13} \mathrm{C}$ ) in low-mass AGB stars (Busso et al. 1999). As shown by Schlattl et al. (2001), in initially metal-free LMSs, the low entropy barrier between the helium- and hydrogen-rich layers enables a penetration of the helium-flash-driven convective zone into the inner tail of the extinguishing H-burning shell. As a consequence, protons are mixed into high temperature regions, triggering an $\mathrm{H}$-burning runaway. The subsequent dredge-up of matter processed by $\mathrm{He}$ and $\mathrm{H}$ burning enriches the surface with large amounts of helium, carbon and nitrogen. Large $[\mathrm{C} / \mathrm{Fe}]$ and $[\mathrm{N} / \mathrm{Fe}]$ abundance ratios are precisely observed in very metal-poor stars (e.g. $[\mathrm{C} / \mathrm{Fe}]$ enhanced by up to 2.0 dex for $[\mathrm{Fe} / \mathrm{H}]<-2.5$; Rossi et al. 1999; see also Chieffi et al. 2001). The possibility (1) is however the more simple and direct explanation for $\mathrm{HC}$-stars in the thick disk.

A very recent result may highlight the issue in favor of possibility (3): new stellar models with masses ranging between 4 and $8 M_{\odot}, Z=0$ and $Y=0.23$ were published by Chieffi et al. (2001). In models with a mass larger than $6 M_{\odot}$, the second dredge-up is able to raise the $\mathrm{CNO}$ abundance in the envelope enough to allow a normal AGB evolution with TPs and TDU. In models of lower mass, the authors find efficient convection associated successively with a He-flash and a $\mathrm{H}$-flash, resulting in carbon abundance in the envelope rising to a level high enough to lead to further evolution similar to that of more metal-rich AGB stars. These population III stars now became white dwarfs provide an important source of primary carbon and nitrogen, which would imply a major revision of the history of chemical evolution in the early Galaxy. Our HC-giants, members of the thick disk, may have received part of this material. Enrichment in s-process elements also takes place in population III AGB stars (Goriely \& Siess 2001).

In possibility (2), the HC-giants could have been halted in their ascent of the AGB by mass loss, leaving only a tiny envelope around a $0.5-0.6 M_{\odot}$-core. This scenario can actually be invoked for faint early HC-stars $\left(-3.5 \lesssim M_{\text {bol }} \lesssim-1\right)$, as descendants of low initial mass stars. Brighter $\left(-3.0 \lesssim M_{\text {bol }} \lesssim-4\right)$ HC5-giants $\left(M \simeq 0.55 M_{\odot}\right)$ and similar CV1-stars could be the remnants of part of higher initial mass stars $\left(\gtrsim 1.1 M_{\odot}\right)$ of the thin disk population. The effect of mass loss is still observed in low mass giants $\left(M \simeq 0.5 M_{\odot}\right)$ like the underluminous CV5-CV6 objects $\left(T_{\text {eff }} \leq 2700 \mathrm{~K}\right)$ of Sample 3 in Bergeat et al. (1998), which are about 1.4 mag fainter than the other cool CV-stars (such underluminous stars are also observed in the LMC).

The underluminous CV5-giants might be objects observed at $M_{\text {bol }} \simeq-3.5 \pm 0.3$ at their luminosity minimum in the interpulse phase (e.g. the evolutionary tracks from: Lattanzio 1987; Sackmann et al. 1993; Steffen et al. 1998; and Ford \& Neufeld 2001). The interpulse period may reach up to $\simeq 2 \times 10^{5}$ Yr for a $M_{\mathrm{c}} \simeq 0.5$ core (e.g. Marigo et al. 1996). The time 
interval spent by the star close to the luminosity minimum is only a small fraction of this period, which could explain why underluminous CV5 and CV6-stars are so few (2\% of our CV-sample). The low (but physically acceptable) pulsation masses derived $\left(\simeq 0.5-0.6 M_{\odot}\right.$, similar to those obtained for the HC5- and CV1-giants) may alternatively suggest that we are observing nearly stripped-off cores with only a tiny envelope of less than $0.1 M_{\odot}$. In such a case, having suffered their last thermal pulse, they may be transiting leftward in the HR diagram, on their track toward the white dwarf region. With further envelope thinning, increasing effective temperatures at nearly constant luminosity are predicted (e.g. the above references). At least part of the HC5- and CV1-giants could thus have the underluminous CV5-stars as progenitors (evolution from $T_{\text {eff }} \simeq 2650 \mathrm{~K}$ up to $3300-3500 \mathrm{~K}$ at nearly constant luminosity $-3.3 \geq M_{\text {bol }} \geq-3.7$ ). In the mass-luminosity diagram (Fig. 4), both objects do populate the lower left corner with $M / M_{\odot}<1$.

Girardi et al (2000) published a diagram showing the relation between the final masses (white dwarfs) and the corresponding initial masses, from both empirical and theoretical origin (their Fig. 4, p. 380). Final masses in the $M \simeq 0.5-$ $0.7 M_{\odot}$ range have progenitors of less than $3 M_{\odot}$ while more massive white dwarfs $\left(M \simeq 0.8-1.1 M_{\odot}\right)$ should be produced by stars initially in the $\simeq 3.5-5.5 M_{\odot}$ interval. For objects having become carbon giants, our results in Table 1 suggest that the boundary between both categories might lie at CV5 with $\left\langle M / M_{\odot}\right\rangle \simeq(2.7 \pm 0.5)$.

The mean stellar density is continuously decreasing along the photometric sequence from HC5 to CV6 at least, from a few $10^{-7}$ to slightly less than $10^{-8}$, in solar units. The surface gravity is nearly constant along the photometric sequence (i.e. along evolutionary tracks) with a mean value of about $g=(5.1 \pm 0.7) \times 10^{-3}$ SI or $\simeq 0.5$ CGS $(\log g \simeq-0.3 \pm 0.1)$. For stars earlier than HC5, an increase to about $\log g \simeq 0$ (or -0.1 ) at $4000 \mathrm{~K}$ is indicated. Both values are in good agreement with the $\log g$ vs. $T_{\text {eff }}$ diagram of Hill et al. (2000, their Fig. 6) for carbon-enriched metal-poor stars. The LPV data roughly obey $P \propto\left(R / R_{\odot}\right)^{1 / 2}$ for both modes, like simple oscillators. With theoretical PMR like $P=Q\left(R / R_{\odot}\right)^{1.5}\left(M / M_{\odot}\right)^{-0.5}$ and $Q$ constant or slowly variable, one obtains $M \propto R^{2}$ or nearly so. We found about $\left(L / L_{\odot}\right) \propto\left(M / M_{\odot}\right)^{0.5}$, and thus $\left(L / L_{\odot}\right) \propto\left(R / R_{\odot}\right)$ or nearly so with an exponent slightly larger than unity. The uniform surface gravity in Table 1 is thus a direct consequence of internal structure and shape of the evolutionary tracks in the HR diagram.

We have attempted to build a global scheme including all of the derived data and many studied objects. Some speculative considerations were of course needed in this discussion, but the authors feel comforted by the consistency and overall agreement of the masses and luminosities, with what can be learnt from other sources (both theory and observations). The lowest effective temperatures $\left(T_{\text {eff }} \leq 2400 \mathrm{~K}\right)$ are not reproduced by evolutionary models. This is not surprising since detailed model atmospheres with extensive opacities (gas + dust) are needed to achieve that, but luminosities are practically not affected. For Milky Way carbon giants, the accuracy of luminosities, photospheric radii and inferred pulsation masses will certainly be strongly improved by future astrometric space missions. Meanwhile, many additional angular diameters (and time variations) will become available from the Very Large Telescope Interferometer (VLTI).

Acknowledgements. Valuable suggestions from the referee Dr. Maurizio Busso are gratefully acknowledged.

\section{References}

Abia, C., \& Isern, J. 1996, ApJ, 460, 443

Alksnis, A., Balklavs, A., Dzervitis, U., \& Eglitis, I. 1998, A\&A, 338, 209

Barnbaum, C., Kastner, J. H., \& Zuckerman, B. 1991, AJ, 102, 289

Barthès, D. 1998, A\&A, 333, 647

Bergeat, J. 2002, in preparation

Bergeat, J., Knapik, A., \& Rutily, B. 1998, A\&A, 332, L53

Bergeat, J., Knapik, A., \& Rutily, B. 2001, A\&A, 369, 178 (Paper I)

Bergeat, J., Knapik, A., \& Rutily, B. 2002a, A\&A, 385, 94 (Paper II)

Bergeat, J., Knapik, A., \& Rutily, B. 2002b, A\&A, 390, 967 (Paper III)

Busso, M., Gallino, R., \& Wasserburg, G. J. 1999, ARA\&A, 37, 239

Chieffi, A., Domínguez, I., Limongi, M., \& Straniero, O. 2001, ApJ, 554,1159

Claussen, M. J., Kleinmann, S. G., Joyce, R. R., \& Jura, M. 1987, ApJS, 65, 385

Dominy, J. F. 1984, ApJS, 55, 27

ESA 1997, The HIPPARCOS Catalogue, ESA SP-1200 (ESA)

Feast, M. W. 1984, MNRAS, 211, 51

Feast, M. W., \& Whitelock, P. A. 1992, MNRAS, 259, 6

Feast, M. W., Glass, I. S., Whitelock, P. A., \& Catchpole, R. M. 1989, MNRAS, 241, 375

Ford, K. E. S., \& Neufeld, D. A. 2001, ApJ, 557, L113

Fox, M. W., \& Wood, P. R. 1982, ApJ, 259, 198

Girardi, L., Bressan, A., Bertelli, G., \& Chiosi, C. 2000, A\&AS, 141, 371

Glass, I. S., \& Lloyd Evans, T. 1981, Nature, 291, 303

Gorely, S., \& Siess, L. 2001, A\&A, 378, L25

Groenewegen, M. A. T., \& de Jong, T. 1994, A\&A, 288, 782

Groenewegen, M. A. T., \& Whitelock, P. A. 1996, MNRAS, 281, 1347

Haniff, C. A., Scholtz, M., \& Tuthill, P. G. 1995, MNRAS, 276, 640

Hartwick, F. D. A., \& Cowley, A. P. 1985, AJ, 90, 2244

Hartwick, F. D. A., \& Cowley, A. P. 1988, ApJ, 334, 135

Hill, V., Barbuy, B., Spite, M., et al. 2000, A\&A, 353, 557

Iben, I., \& Renzini, A. 1983, ARA\&A, 21, 27

Kholopov, P. N., Samus, N. N., Frolov, M. S., et al. 1985, General Catalogue of Variable Stars (Nauka Publishing House, Moscow) (GCVS). New edition as an electronic version at the Sternberg Institute in Moskow (ftp sai.msu.su with anonymous as user and email-address as pw).

Kahane, C., Dufour, E., Busso, M., et al. 2000, A\&A, 357, 669

Kipper, T., \& Jørgensen, U. G. 1994, A\&A, 290, 148

Kipper, T., Jørgensen, U. G., Klochkova, V. G., \& Panchuk, V. E. 1996, A\&A, 306, 489

Kiss, L. L., Szatmáry, K., Cadmus, Jr. R. R., \& Mattei, J. A. 1999, A\&A, 346, 542

Knapik, A., Bergeat, J., \& Rutily, B. 1999, A\&A, 344, 263

Lambert, D. L., Gustafsson, B., Erikson, K., \& Hinkle, K. H. 1986, ApJS, 62, 373

Lattanzio, J. C. 1987, ApJ, 313, L15

Marigo, P., Bressan, A., \& Chiosi, C. 1996, A\&A, 313, 545

Marigo, P., Girardi, L., \& Bressan, A. 1999, A\&A, 344, 123

McClure, R. D. 1989, in Evolution of Peculiar Red Giant Stars, ed. H. R. Johnson, \& B. Zuckerman (Cambridge University Press, Cambridge), IAU Colloq., 106, 196 
McClure, R. D. 1997a, PASP, 109, 256

McClure, R. D., \& Woodsworth, A. W. 1990, ApJ, 352, 709

Norris, J. E., Ryan, S. G., \& Beers, T. C. 2001, ApJ, 561, 1034

Percy, J. R., Wilson, J. B., \& Henry, G. W. 2001, PASP, 113, 983

Prochaska, J. X., Naumov, S. O., Carney, B. W., McWilliam, A., \& Wolfe, A. M. 2000, AJ, 120, 2513

Reid, I. N., Hughes, S. M. G., \& Glass, I. S. 1995, MNRAS, 275, 331

Rossi, S., Beers, T. C., \& Sneden, C. 1999, in The Third Stromlo Symposium: The Galactic Halo, ASP Conf. Ser., 165, 264

Sackmann, I.-J., Boothroyd, A. I., \& Kraemer, A. E. 1993, ApJ, 418, 457

Scalo, J. M. 1976, ApJ, 206, 474

Schlattl, H., Cassini, S., Salaris, M., \& Weiss, A. 2001, ApJ, 559, 1082

Smith, H. Jr., \& Eichhorn, H. 1996, MNRAS, 281, 211

Steffen, M., Szczerba, R., \& Schoenbener, D. 1998, A\&A, 337, 149

Thronson, Jr. H. A., Latter, W. B., Black, J. H., Bally, J., \& Hacking, P. 1987, ApJ, 322, 770

Tuchman, Y., Sack, N., \& Barkat, Z. 1979, ApJ, 234, 217
Van Eck, S., Jorissen, A., Udry, S., Mayor, M., \& Pernier, B. 1998, A\&A, 329, 971

van Leeuven, F., Feast, M. W., Whitelock, P. A., \& Yudin, B. 1997, MNRAS, 287, 955

Vanture, A. D. 1987, AJ, 104, 1986

Westerlund, B. E., Azzopardi, M., Breysacher, J., \& Rebeirot, E. 1991a, A\&A, 244, 367

Westerlund, B. E., Azzopardi, M., Breysacher, J., \& Rebeirot, E. 1995, A\&A, 303, 107

Wood, P. R. 1974, ApJ, 190, 609

Wood, P. R. 1990, in From Miras to Planetary Nebulae, ed. M. O. Mennessier, \& A. Omont

Wood, P. R., Bessell, M. S., \& Fox, M. W. 1983, ApJ, 272, 99

Wood, P. R., \& Sebo, K. M. 1996, MNRAS, 282, 958

Ya'ari, A. 1999, private communication

Ya'ari, A., \& Tuchman, Y. 1996, ApJ, 456, 350

Ya'ari, A., \& Tuchman, Y. 1999, ApJ, 514, L35

Zuckerman, B., \& Dyck, H. M. 1989, A\&A, 209, 119 